M ddl e- aged Japanese women ar e resi st ant to obesi ty-rel at ed met abol i c abnor mal i ti es

\begin{tabular}{|l|l|}
\hline 著者 & $\begin{array}{l}\text { Sakur ai Nasar u, Takamur a Toshi nar i, M ur a } \\
\text { Kat suyuki, Kaneko Shui chi, Nakagawa H deaki }\end{array}$ \\
\hline $\begin{array}{l}\text { j our nal or } \\
\text { publ i cat i on t i t l e }\end{array}$ & Net abol i sm Cl i ni cal and Exper i ment al \\
\hline vol une & 58 \\
\hline nunber & 4 \\
\hline page r ange & $456-459$ \\
\hline year & $2009-04-01$ \\
\hline URL & ht t p: //hdl . handl e. net /2297/17359 \\
\hline
\end{tabular}




\section{Middle-aged Japanese women are resistant to obesity-related metabolic abnormalities}

Masaru Sakurai $^{1,2}$, Toshinari Takamura ${ }^{1}$, Katsuyuki Miura ${ }^{3}$, Shuichi Kaneko ${ }^{1}$, Hideaki Nakagawa ${ }^{2}$

${ }^{1}$ Department of Disease Control and Homeostasis, Kanazawa University Graduate School of Medical Science, Kanazawa, Japan

${ }^{2}$ Department of Epidemiology and Public Health, Kanazawa Medical University, Ishikawa, Japan

${ }^{3}$ Department of Health Science, Shiga University of Medical Science, Otsu, Japan

Address correspondence and reprint request to: Dr. Toshinari Takamura, Department of Disease Control and Homeostasis, Kanazawa University Graduate School of Medical Science, 13-1 Takara-machi, Kanazawa, Ishikawa 920-8641, Japan E-mail: ttakamura@m-kanazawa.jp 


\section{Abstract}

We attempted to determine gender differences in obesity-related metabolic abnormalities in a relatively large middle-aged Japanese population. The study population consisted of 2,935 men and 1,622 women who were 35-59 years old. Metabolic abnormalities were determined using the Japanese criteria for metabolic syndrome, and we evaluated the number of metabolic abnormalities discriminated by waist circumference. In men, the mean number of metabolic abnormalities increased as the waist circumference increased. In women, although the mean number of metabolic abnormalities increased as the waist circumference increased, the mean number was $<1$ even in those with a waist circumference $\geq 95 \mathrm{~cm}$. According to the receiver-operator characteristic (ROC) curve, the cutoff levels yielding the maximal sensitivity plus specificity for predicting the prevalence of one or more obesity-related metabolic abnormalities were $80 \mathrm{~cm}$ in men and $73 \mathrm{~cm}$ in women. However, the positive predictive value was as low as $28.8 \%$ in men and $7.1 \%$ in women, which may not be suitable for a screening test, especially in women. Middle-aged Japanese women seem to be resistant to obesity-induced metabolic abnormalities, and waist circumference would not effectively predict the existence of metabolic syndrome. In setting the cutoff points in guidelines, a greater emphasis should be placed on the absolute risk of having abnormalities or diseases. 


\section{Introduction}

Cutoff points for waist circumference have been proposed for each racial/ethnic group in diagnosing abdominal obesity and metabolic syndrome [1]. However, the Japanese cutoff points for waist circumference are still controversial. The Japanese cutoff points proposed by Japan Society of Study for Obesity were derived from a $100-\mathrm{cm}^{2}$ area for visceral fat at the umbilical level on computed tomography imaging and are unique in that the value for women $(90 \mathrm{~cm})$ is larger than that for men $(85 \mathrm{~cm})[2,3]$. On the other hand, International Diabetes Federation (IDF) propose to use the cutoff point of waist circumference $90 \mathrm{~cm}$ for men and $80 \mathrm{~cm}$ for women [1], and some reports proposed lowering the cutoff point of waist circumference for Japanese women [4,5]. However, previous reports did not pay attention to the absolute risk of the accumulation of metabolic abnormalities in Japanese women. We attempted to determine gender differences in the accumulation of metabolic abnormalities in relation to obesity in a relatively large-scale middle-aged Japanese population. 


\section{Research design and methods}

The study population consisted of 4,557 Japanese employees (2,935 men and 1,622 women) of a metal-products factory who were aged 35-59 years. Detailed information on this study population has been provided elsewhere [6,7]. The physical examinations for this analysis were held in 1996. Anthropometric markers including waist circumference and blood pressure were measured, and venous blood samples after an overnight fast were withdrawn from each subject during a routine annual medical checkup. Three obesity-related abnormalities, high blood pressure, dyslipidemia, and high fasting plasma glucose, were defined by the Japanese guidelines of metabolic syndrome [3]. High blood pressure was defined as a systolic blood pressure $\geq 130 \mathrm{mmHg}$ or a diastolic blood pressure $\geq 85 \mathrm{mmHg}$. Dyslipidemia was defined as serum triglycerides $\geq 150 \mathrm{mg} / \mathrm{dl}$ or HDL-cholesterol $\leq 40 \mathrm{mg} / \mathrm{dl}$, and high fasting plasma glucose was defined as a fasting plasma glucose $\geq 110 \mathrm{mg} / \mathrm{dl}$. We evaluated the number of obesity-related abnormalities discriminated by waist circumference and body mass index (BMI). We plotted receiver-operator characteristic (ROC) curves for waist circumference to predict one or more obesity-related metabolic abnormalities and calculated sensitivity, specificity, and positive predictive value. 


\section{Results}

The participants had a mean age of 45.5 years for men and 45.3 years for women, a mean BMI of $23.3 \mathrm{~kg} / \mathrm{m}^{2}$ for men and $22.6 \mathrm{~kg} / \mathrm{m}^{2}$ for women, and a mean waist circumference of $80.1 \mathrm{~cm}$ for men and $72.7 \mathrm{~cm}$ for women.

We evaluated the association between waist circumference or BMI and the mean number of obesity-related abnormalities (Fig. 1). In men, the mean number of abnormalities increased as waist circumference or BMI increased. When the waist circumference was $85 \mathrm{~cm}$, which is the cutoff point for the diagnosis of metabolic syndrome in Japanese men [2], the mean number of complicated metabolic abnormalities was approximately 1. In women, although the mean number of abnormalities increased as the waist circumference increased, it was $<1$ even in women with a waist circumference $\geq 95 \mathrm{~cm}$ or BMI $\geq 30$ $\mathrm{kg} / \mathrm{m}^{2}$.

The prevalence of one or more obesity-related abnormalities was $50 \%$ in men and $21 \%$ in women. According to the ROC curve, the area under the curve was higher for men (0.675; 95\% CI, 0.655-0.694) than for women (0.627; 95\% CI, 0.596-0.657) (Fig. 2). The cutoff levels yielding the maximal sensitivity plus specificity for predicting the prevalence of one or more obesity-related abnormalities were $80 \mathrm{~cm}$ in men (sensitivity 0.59 , specificity 0.69 ) and $73 \mathrm{~cm}$ in women (sensitivity 0.55 , specificity 0.64 ). However, the positive predictive value was as low as $28.8 \%$ in men and $7.1 \%$ in women at these cutoff points. 


\section{Discussion}

Hara et al. [4] and Ohkubo et al. [5] proposed a cutoff point regarding waist circumference for detecting metabolic syndrome or insulin resistance in Japanese based on relatively small, older, and somewhat higher-risk populations. Both reports proposed lowering the cutoff point of waist circumference for Japanese women to $76 \mathrm{~cm}$. We found that a similar cutoff point of $73 \mathrm{~cm}$ for women provided the highest sensitivity and specificity for detecting metabolic syndrome as was recently observed $[4,5]$.

Theoretically, sensitivity and specificity (therefore, the ROC curves) are not affected by the prevalence of the detected disease in various populations. However, the positive predictive value was as low as $7.1 \%$ in our population of middle-aged Japanese women, which means that only $7.1 \%$ of women with waist circumference $73 \mathrm{~cm}$ or over had the accumulation of metabolic abnormalities. Therefore this cutoff point may not be suitable for a screening test in women. Two previous reports from Japan did not give the positive predictive values for their cutoff points. The low positive predictive value was caused by the low prevalence of metabolic abnormalities in middle-aged Japanese women, and possibly the relatively higher resistance of Japanese women than men to obesity-induced metabolic abnormalities. As proposed in previous reports [4,5], lower cutoff points for waist circumference might detect more people with metabolic abnormalities with high sensitivity. However, in a population with a low prevalence of metabolic abnormalities, lower cutoff points would also result in a greater proportion of false-positives, with more healthy women in particular being screened as abnormal. Furthermore, because the mean number of complicated metabolic abnormalities was lower than 1 even in those with a waist circumference $\geq 95 \mathrm{~cm}$ and the area under the ROC curve was lower in women than 
in men, waist circumference would not effectively predict the existence of metabolic syndrome in Japanese women.

In this study, similar to previous reports [2,4,5,8], cutoff points of waist circumference were proposed using ROC curves for predicting the metabolic abnormalities. However, abdominal obesity is important in metabolic syndrome because it has been linked to the development of cardiovascular disease. Further investigations are needed to evaluate the association between waist circumference and future incidence of cardiovascular events to establish an appropriate cutoff point for waist circumference. Another limitation was that the participants of this study did not include older people and might be healthier than general Japanese people because they were identified at a work place. Similar analyses are needed in older Asian populations.

In conclusion, middle-aged Japanese women seem to be resistant to obesity-related metabolic abnormalities, and waist circumference would not effectively predict the existence of metabolic syndrome. In setting cutoff points in guidelines, a greater emphasis should be placed on the absolute risk of having abnormalities or diseases. 


\section{References}

[1] Arberti KG, Zimmet P, Shaw J for the IDF Epidemiology Task Force Consensus Group. The metabolic syndrome-a new worldwide definition. Lancet 2005;366:1059-62.

[2] The Examination Committee of Criteria for “Obesity Disease” in Japan, Japan Society for Study of Obesity. New criteria for 'obesity disease’ in Japan. Circulation J 2002;66:987-92.

[3] Oda E, Watanabe K. Japanese criteria of metabolic syndrome. Circ J 2006;70:364.

[4] Hara K, Matsushita Y, Horikoshi M, Yoshiike N, Yokoyama T, Tanaka H, et al. A proposal for the cutoff point of waist circumference for the diagnosis of metabolic syndrome in the Japanese population. Diabetes Care 2006;29:1123-4.

[5] Ohkubo T, Kikuya M, Asayama K. A proposal for the cutoff point of waist circumference for the diagnosis of metabolic syndrome in the Japanese population. Diabetes Care 2006;29:1986-7.

[6] Sakurai M, Miura K, Takamura T, Ota T, Ishizaki M, Morikawa Y, et al. Gender differences in the association between anthropometric indices of obesity and blood pressure in Japanese. Hypertens Res 2006;29:75-80.

[7] Morikawa Y, Nakagawa H, Ishizaki M, Tabata M, Nishijo M, Miura K, et al. Ten-year follow-up study on the relation between the development of non-insulin dependent diabetes mellitus and occupation. Am J Ind Med 1997;31:80-4.

[8] Ko GT, Chan JC, Cockram CS, Woo J. Prediction of hypertension, diabetes, dyslipidaemia or albuminuria using simple anthropometric indexes in Hong Kong Chinese. Int J Obes Relat Metab Disord 1999;23:1136-42. 


\section{Figure legends}

Fig. 1. The mean number of obesity-related abnormalities discriminated by waist circumference or body mass index in men ( $\mathbf{a})$ and women (०).

Obesity-related abnormalities included hypertension, dyslipidemia, and glucose intolerance. The horizontal dotted line shows a mean number of obesity-related abnormalities of 1.0. Data are presented as means \pm standard error.

Fig. 2. The predictive performance of waist circumference for one or more obesity-related metabolic abnormalities. The receiver operating characteristic curves for one or more obesity-related metabolic abnormalities in men (A) and in women (B) are shown. The sensitivity and specificity of each cutoff point of waist circumference are given in (C). 
Figure 1
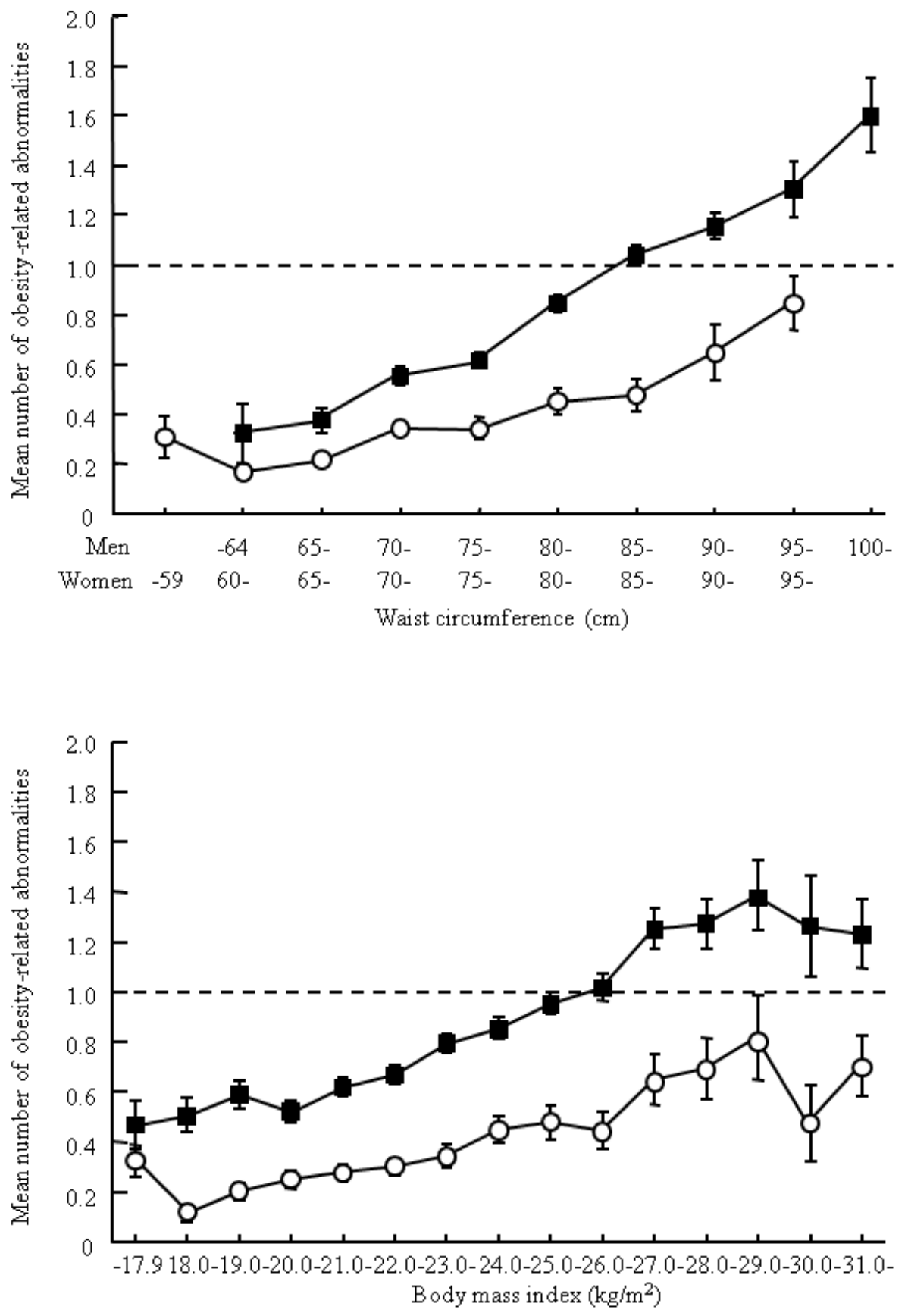
Figure 2

\section{A. Men}

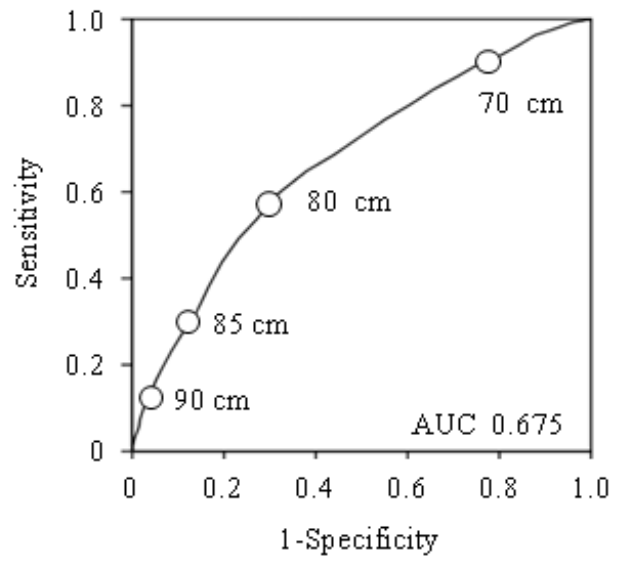

B. Women

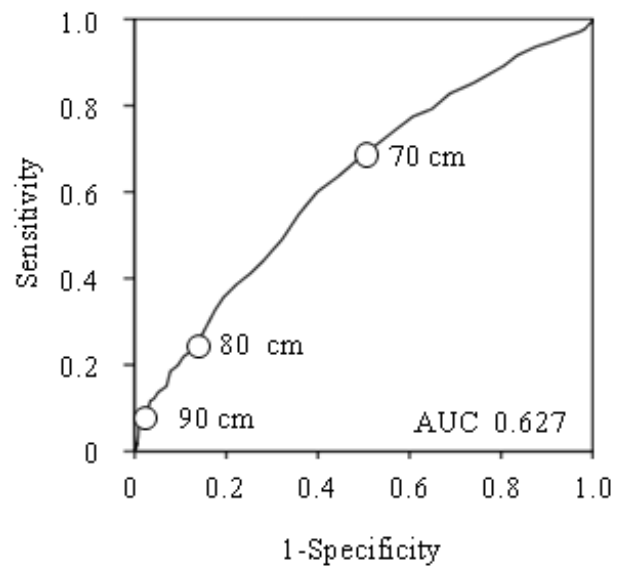

C. Cutoff point for wast circumference

\begin{tabular}{|c|c|c|}
\hline & Sensitivity & Specificity \\
\hline \multicolumn{3}{|l|}{ Men } \\
\hline $70 \mathrm{~cm}$ & 0.94 & 0.16 \\
\hline $80 \mathrm{~cm}$ & 0.59 & 0.69 \\
\hline $85 \mathrm{~cm}$ & 0.32 & 0.86 \\
\hline $90 \mathrm{~cm}$ & 0.13 & 0.96 \\
\hline \multicolumn{3}{|l|}{ Women } \\
\hline $70 \mathrm{~cm}$ & 0.67 & 0.52 \\
\hline $73 \mathrm{~cm}$ & 0.55 & 0.64 \\
\hline $80 \mathrm{~cm}$ & 0.27 & 0.85 \\
\hline $90 \mathrm{~cm}$ & 0.09 & 0.98 \\
\hline
\end{tabular}

